data in CHD cases will be a productive means of identifying causative genes. In this study we have undertaken various refinement steps to narrow down potentially causative candidate gene/s within deleted (DEL) and duplicated (DUP) CNV regions that have been previously shown to be highly associated with non-syndromic CHD patients. Firstly, we have generated case DEL and DUP CNV lists. This was done by updating a published meta-analysis study (our group have contributed to this study) though utilisation of DECIPHER, ISCA, ECARUCA databases and published studies, using different key terms to identify further non-syndromic CHD patients. We then utilised BedTools to compare these case lists with the corresponding control CNV lists generated by using controls from published literature, DECIPHER, the Database of Genomic Variants and the 1000 Genome Phase 3 CNVs. The resulting unique cases $\mathrm{CNV}$ regions were annotated and compared against an in-house list of candidate genes (containing novel or rare variants) generated from an exome data analysis of 850 Tetralogy of Fallot (ToF) patients. Genes were further prioritised based on whether they have already an assigned human phenotype, on their ExAC CNV scores, probability of haploinsufficiency $(\mathrm{pHI})$ and loss of function (LoF) intolerance scores. Initially, we have identified 10586 genes for which 1986 genes are present in both DEL and DUP CNV regions, 2772 genes are unique in DUP and 3842 genes unique in DEL regions (Figure 1). Further analysis of genes in DEL regions and genes present in both types of $\mathrm{CNVs}$ revealed that 1,150/3,842 genes and 588/1,986 genes respectively have LoF variants in our ToF exome data. Additional filtering with $\mathrm{pHI}$ and pLI scores resulted in 57 genes collectively. This is an on-going work and our plan is to design a next-generation sequencing panel to screen our final candidate gene list in an additional 2000 CHD cases. We will focus on the most promising candidate gene emerging from the discovery experiment to perform functional work. Our experimental strategy will vary depending on what is known about the gene, and whether its involved or not in any well-known signalling pathway during heart development such as the Wnt and VEGF pathways.

\section{LOX-1-SPECIFIC AFFIMERS BLOCK OXLDL ACCUMULATION IN VITRO}

Jonathan De Siqueira*, Izma Abdul Zani, David Russell, Stephen Wheatcroft, Darren Tomlinson, Shervanthi Homer-Vanniasinkam, Sreenivasan Ponnambalam. University of Leeds

\subsection{6/heartjnl-2017-311726.162}

Introduction Lectin-like oxidised low density lipoprotein receptor 1 (LOX-1, SREC-1, OLR1) is a class E scavenger which binds oxidised low density lipoproteins (oxLDL). It is expressed on endothelial cells, macrophages and smooth muscle cells, where it has a major role in sensing oxLDL, in the triggering of cellular dysfunction and in contribution to atherosclerosis. LOX-1 is thus an important target for the prevention and/or treatment of atherosclerosis. Affimers are synthetic protein scaffolds containing two hypervariable amino acid loops which enable recognition of a wide range of molecules. We aimed to investigate whether affimers specific for LOX-1 could be used to target oxLDL recognition and uptake using an in vitro cellular model.

Methods Human embryonic kidney (HEK293T) and porcine aortic endothelial cells (PAEC) with tetracycline-inducible protein expression were engineered to expressed human LOX-1FLAG construct. Isolated clonal lines for each cell type was checked using RT-PCR, immunoblotting and immunofluorescence microscopy. After overnight incubation with tetracycline ( $1 \mathrm{microgram} / \mathrm{ml}$ ), cells were starved and incubated with $=1,1^{\text {c }}$ Dioctadecyl-3,3,3',3'-Tetramethylindocarbocyanine Perchlorate

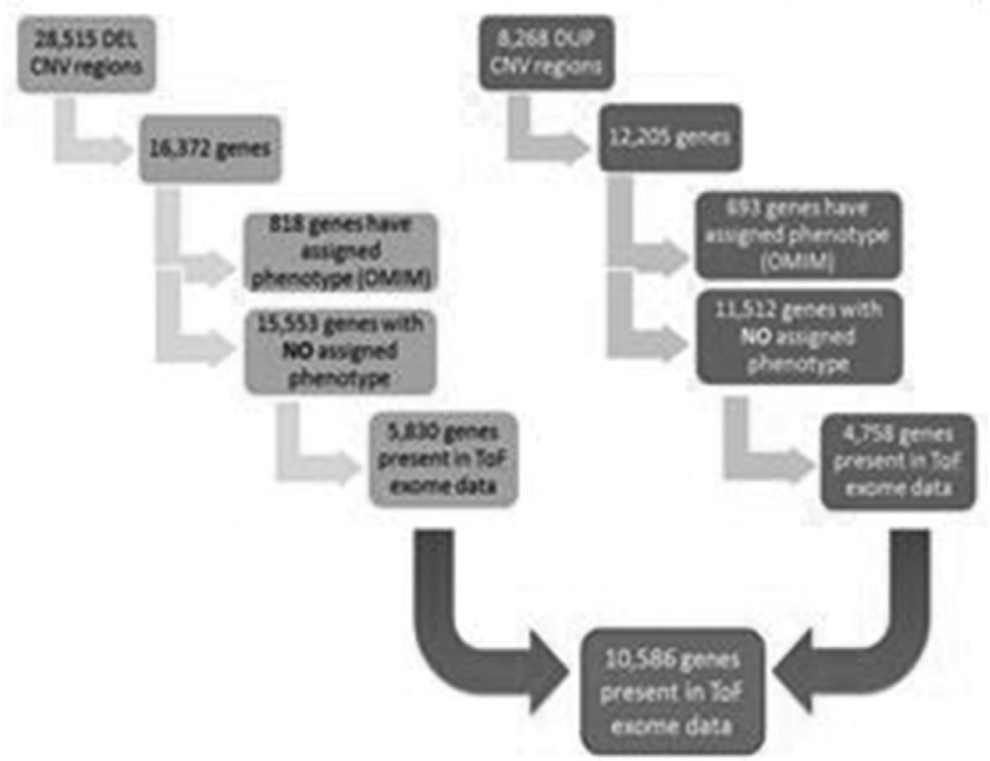

Abstract 162 Figure 1 Flowchart of the initial filtering of CNV regions to genes. 
A

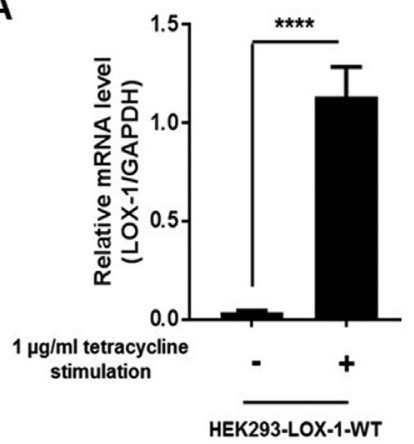

B

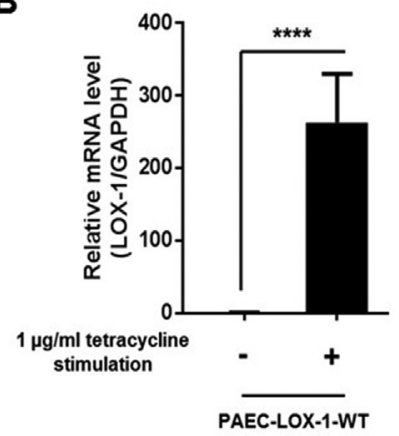

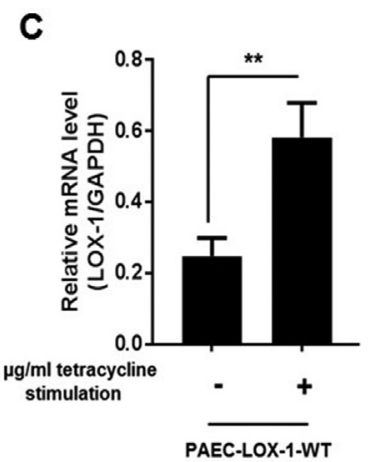

Abstract 163 Figure 1 Relative mRNA levels of: A) Human LOX-1 in HEKs, B) Human LOX-1 in PAECs and C) Porcine LOX-1 in PAECs

A
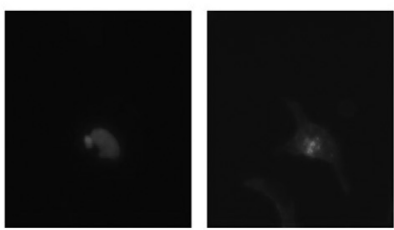

B

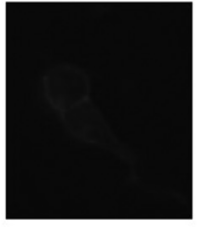

0

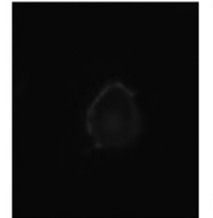

5
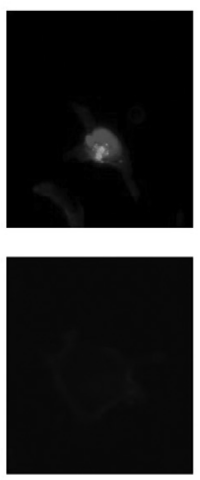

50

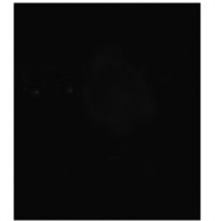

500

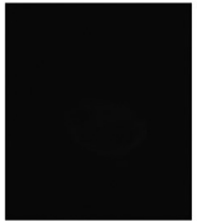

5000

Abstract 163 Figure 2 Immunofluorescence Microscopy of HEK293T after induction with 1microgram/ml Tetracycline. A) Blue (DAPI) staining of nucleus and green (anti-FLAG) staining of FLAG-tagged LOX-1 B) Affimer effects on oxLDL (red) uptake.

(DiI)-labelled oxLDL (10 microgram/ml) for $30 \mathrm{~min}$. Cells were fixed and imaged to characterise the uptake of OxLDL. Blocking experiments using 5 different affimers, was carried out by adding affimer prior to incubation with DiI-oxLDL. Effects on oxLDL binding and cellular accumulation was evaluated using fluorescence microscopy.

Results The levels of LOX-1-FLAG mRNA were significantly raised $(p<0.001)$ in both cell types in response to tetracycline. Porcine endothelial cells also had significant levels of endogenous native porcine LOX-1(Figure 1). The presence of human LOX-1-FLAG protein was confirmed by immunofluorescence microscopy (Figure 2a). Incubation with oxLDL demonstrated tetracycline-dependent labelled lipid particle uptake in HEK293T LOX-1-FLAG expressing cells. In contrast, oxLDL uptake was not significantly raised compared to uninduced baseline in PAEC LOX-1-FLAG expressing cells. Incubation with LOX-1 binding affimers led to a decrease in oxLDL uptake in both cell lines after induction of LOX-1-FLAG expression (Figure 2b).

Conclusion We have successfully constructed two mammalian cell lines with a stable, inducible LOX-1 expression system. This has allowed us to demonstrate LOX-1-dependent oxLDL uptake. However, such expression is dependent on negligible expression of other scavenger receptors that mediate oxLDL binding and/or uptake. The use of LOX-1-specific affimers show that oxLDL binding and/or uptake is significantly decreased in both cell lines. Future experiments aim to determine the effects of such LOX-1-specific affimers in primary vascular cells and animal models in the context of atherosclerosis and vascular disease.

\section{ABSTRACT WITHDRAWN}

\section{HUMAN ATHEROSCLEROSIS IS CHARACTERISED BY OXIDATIVE DNA DAMAGE DUE TO DEFECTIVE BASE EXCISION REPAIR}

Aarti Shah*, Kelly Gray, Nichola Figg, Martin Bennett. University of Cambridge

\subsection{6/heartjnl-2017-311726.163}

Rationale Human atherosclerotic plaques show extensive oxidative DNA damage in vascular smooth muscle cells (VSMCs) and macrophages, including accumulation of 8-oxo-7,8-dihydroguanine (8-oxoG), the most abundant DNA base lesion on oxidative exposure. 8 -oxoG is repaired by base excision repair (BER) mediated by DNA glycosylases, including the specific non-redundant 8-oxoguanine DNA glycosylase-1 (OGG1). OGG1 activity is regulated by acetylation through the p300 acetyltransferase. However, the role of oxidative damage in VSMC function and the regulation of OGG1 in atherosclerosis are unknown.

Methodology We stably expressed OGG1 or the ${ }^{\mathrm{K} 338 / \mathrm{K} 341} \mathrm{OGG} 1$ acetylation mutant in rat VSMCs in vitro, and treated cells with oxidative stress. The functional effects of OGG1 in vivo were 